\title{
LA PROTECTION SOCIALE DES TRAVAILLEURS MIGRANTS : UNE ANALYSE MULTI-NIVEAUX DES REGIMES DE SECURITE SOCIALE
}

\author{
PROTECCIÓN SOCIAL DE LOS TRABAJADORES MIGRANTES: UN \\ ANÁLISIS MULTI-NIVEL DE LOS SISTEMAS DE SEGURIDAD \\ SOCIAL
}

\section{ELISA FORNALÉ ${ }^{1}$}

Professeure boursière FNS, World Trade Institute, Université de Berne.

\footnotetext{
${ }^{1}$ L'auteur souhaite remercier le Prof. Cristina Sánchez-Rodas Navarro qui a organisé le colloque et avec qui elle a eu le privilège de collaborer pendant la mise en œuvre du projet R_eMigra. En outre, l'auteur souhaite remercier M.A. Lisa Salcedo Pfeiffer, spécialiste en migrations et développement, pour la relecture attentive et ses commentaires sur cet article. La recherche conduite pour réaliser cet article a été financée par le Fonds National Suisse de la recherche scientifique et le programme Marie Curie (FP7).
}

e-Revista Internacional de la Protección Social, ISNN 2445-3269. 2017, Vol. II, No 2 


\title{
RESUME
}

L'article vise à développer une étude sur l'évolution de l'accès aux droits sociaux des travailleurs migrants et leur portabilité dans un cadre juridique multi-niveaux. L'article fournira une introduction historique aux règles et aux modalités d'accès à la protection sociale dans le contexte des accords existants, avec une attention particulière portée sur les niveaux bilatéral et régional. L'objectif est de mieux comprendre l'origine des régimes de sécurité sociale et d'identifier les principales caractéristiques de ces modèles. La présentation de pratiques concernant les mécanismes de portabilité des prestations de sécurité sociale contribuera à l'identification des éléments requis afin de promouvoir un système transnational de droits de sécurité sociale.

MOTS CLES: Travailleurs migrants, Sécurité sociale, Portabilité.

\begin{abstract}
The chapter adopts an historical approach in reading the evolution and origins of social rights and their portability for international migrants in two different regimes (bilateral and regional units) reviewing the most important literature on the subject. This chapter relates the concepts of regionalism/bilateralism to contemporary debates on multilevel migration governance to assess their significance considering first the unsolved problem of defining social security regimes, their origins, present and future prospects for the status of social protection, and their place in global order. The paper will then take a closer look at the proliferation of regional/bilateral groupings in terms of the key features of those arrangements. This paper will lay the basis for a study on the emerging models that could guarantee an improved access to specific forms of social protection and portability arrangements.
\end{abstract}

KEYWORDS: Migrant Workers, Social Security, Portability. 


\section{RESUME}

\section{INTRODUCTION}

II. MISE EN CONTEXTE ET DEFINITIONS

III. EVOLUTION HISTORIQUE DU CADRE NORMATIF INTERNATIONAL

IV. LES ACCORDS BILATERAUX

V. L'EVOLUTION DE LA COORDINATION DE LA SECURITE SOCIALE AU NIVEAU REGIONAL

VI. CONCLUSION 


\section{INTRODUCTION}

Le débat autour de l'accès à la protection sociale et à la portabilité des ses prestations pour les travailleurs migrants a connu une évolution 'remarquable' au cours des dernières années ${ }^{2}$. En particulier, la protection sociale des travailleurs migrants à l'intérieur de différents pays est une préoccupation constante qui soulève, au niveau international, des questions d'ordre économique mais également d'ordre social ${ }^{3}$.

Comme souligné par la Conférence internationale du Travail (2011), faire bénéficier les travailleurs migrants de l'égalité de traitement en matière de sécurité sociale constitue une nécessité sociale. En effet, environ 243,7 millions de personnes vivent et travaillent en dehors de leur pays d'origine et, selon les estimations futures, l'ampleur des flux migratoires serait en hausse ${ }^{4}$. Il est de plus en plus admis que le fait de faire bénéficier aux travailleurs migrants; indépendamment qu'ils aient un statut temporaire ou de résident permanent; des mêmes conditions que celles accordées aux travailleurs nationaux, «participe à la lutte contre les inégalités dans une perspective de justice sociale ${ }^{5}$. En outre, l'exclusion des travailleurs migrants des régimes de sécurité sociale nationaux peut entraîner des répercussions négatives sur leur intégration et aussi affecter leur mobilité ${ }^{6}$. Le citoyen étranger risque de se sentir «démuni» face au pouvoir arbitraire de l'Etat et «le caractère incertain et spoliateur des prélèvements obligatoires » qui, pour paraphraser Salin, peuvent générer le sentiment d'être soumis à une tyrannie ${ }^{7}$.

La problématique est très complexe pour différentes raisons et suscite des défis juridiques épineux. Historiquement, l'accès aux prestations sociales relève du droit interne et est régi par le principe de territorialité. Comme souligné par ROBERTS « l'approche traditionnelle considère que les droits à une protection sociale émanent des Etats-nations et sont circonscrits au territoire national $»^{8}$. Dans ce contexte, l'attribution des prestations sociales aux travailleurs migrants est liée aux critères de la résidence habituelle, ou de la nationalité, et à la subsistance d'un lien suffisamment étroit avec le pays concerné. C'est la remise en question de l'unité territoriale du régime de la sécurité sociale qui continue de se heurter à une forte résistance dans le cadre de l'extension de la couverture aux travailleurs migrants.

\footnotetext{
${ }^{2}$ Organisation Internationale du Travail (OIT) (2016), Promouvoir une migration équitable, Conférence Internationale du travail, 105e Session.

${ }^{3}$ Penning, FransetVonk, Gijsbert (eds.) (2015), Research Handbook on European Social Security Law, Cheltenham, Edward Elgar Publishing.

${ }^{4}$ OIT, 2016; AISS (2014), Manuel sur l'extension de la couverture de la sécurité sociale aux travailleurs migrants.

${ }^{5}$ La présente étude n'inclut pas les migrants qui travaillent de façon informelle. Lamarche, Lucie (2012), «Le droit social et les droits sociaux : des outils dissonants pour la régulation sociale dans le contexte du néolibéralisme», dans Roman, Diane (dir.), La justiciabilité des droits sociaux, vecteurs et résistances, Paris, Pedonep, 119-137.

${ }^{6}$ Tout récemment, Holzmann a conduit une étude concernant quatre accords bilatéraux (Autriche-Turquie, Allemagne- Turquie, Belgique-Maroc, France-Maroc) pour explorer l'impact de l'existence ou non d'accords bilatéraux sur la portabilité sur la mobilité de travailleurs migrants. Holzmann, Robert (2016), Bilateral social security agreements and pensions portability: A study of four migrant corridors between EU and non-EU countries, International Social Security Review, vol. 69, 3-4/2016, pp. 109-130.

${ }^{7}$ Salin, Pascal (2014), La Tyrannie Fiscale, Paris, Odile Jacob, p. 10.

${ }^{8}$ Roberts, Simon (2010), Bref historique de la coordination de la sécurité sociale, dans Jorens, Yves, Commission européenne, Direction générale de l'emploi, des affaires sociales et de l'égalité des chances, 50 ans de coordination de la sécurité sociale : passé - présent - futur : rapport de la conférencecélébrant le 50e anniversaire de la coordination européenne de la sécurité sociale : Prague, 7 et 8 mai 2009, pp. 8-29.
}

e-Revista Internacional de la Protección Social, ISNN 2445-3269. 2017, Vol. II, No 2

http://dx.doi.org/10.12795/e-RIPS.2017.i02.04.

Página 21 
Les ressortissants de pays tiers se déplacent «d'un pays à l'autre et par conséquent entre des marchés du travail et des systèmes de sécurité sociale règlementés de façon distincte» ${ }^{9}$. Au fil des années, le besoin de garantir que les étrangers ne perdent pas leurs droits a fait évoluer le cadre normatif avec l'élaboration de différents régimes stratifiés. D'une part, cette profusion de normes et la coexistence de différents régimes (par exemple universels, régionaux et bilatéraux) forme un cadre normatif très diversifié qui peut répondre à une approche plus individuelle dans leur mise en application ${ }^{10}$. D'autre part, l'absence d'une approche globale fait apparaître un décalage entre la situation des citoyens de pays qui ont signé des accords et ceux de nombreux pays où aucun instrument de coordination n'a été mis en place.

L'analyse vise à adresser le problème sous une perspective juridique pour se pencher sur les questions suivantes: Comment les instruments actuels coordonnent-ils les régimes de sécurité sociale? Comment réguler la portabilité de droits sociaux? Existe-il un droit d'exclusion en matière de protection sociale vis-à-vis des travailleurs migrants?

\section{MISE EN CONTEXTE ET DEFINITIONS}

Une précision s'impose au préalable. La «protection sociale» est ici comprise sous deux angles spécifiques. Elle fait référence, d'une part, aux droits garantis -en particulier l'accès à la sécurité sociale ${ }^{11}$ - dans les pays d'origine et de destination, et d'autre part, à la transférabilité ${ }^{12}$ desdits droits entre les pays concernés ${ }^{13}$.

Il est généralement admis que les systèmes de «protection sociale» reposent sur le travail, c'est-à-dire qu'ils sont financés par des impôts et des cotisations proportionnels aux revenus du travailleur ${ }^{14}$. Dans la plupart des pays, le financement de la protection sociale a été nationalisé. Cette nationalisation, «faite au nom de la solidarité», affecte en particulier la situation individuelle du citoyen étranger qui est obligé «d'acheter les mêmes paniers de police» sociale que les nationaux sans pour autant bénéficier automatiquement et pleinement des mêmes prestations et droits sociaux s'il/elle décide, par exemple, de retourner dans son pays d'origine ${ }^{15}$. Comme l'a récemment affirmé l'Association internationale de la sécurité sociale (AISS), «les migrants paient souvent plus qu'ils ne reçoivent, et ce même en ce qui concerne les programmes de sécurité sociale totalement ou partiellement financés par les recettes fiscales, étant donné qu'ils participent à leur financement par le biais des taxes à la consommation» ${ }^{16}$. Le travailleur

\footnotetext{
${ }^{9}$ Sabates-Wheeler, Rachel et Koettls, Johannes (2010), «Les problèmes de fourniture d'une protection sociale aux migrants dans le contexte de l'évolution des flux migratoires», Revue Internationale de Sécurité Sociale, vol. 63, n. 3-4, p. 123-157, p. 125.

${ }^{10}$ AISS, 2014.

${ }^{11}$ Pour la définition de sécurité sociale, voir Sabates-Wheeler et Koettls, 2010, p. 128: «La sécurité sociale s'entend ici comme comprenant les prestations sociales (en grand partie financées par l'impôt) et l'assurance sociale (financée par des cotisations)».

${ }^{12}$ Selon Cruz, 2004, la transférabilité «est la capacité de préserver, maintenir et transférer des droits acquis en matière de sécurité sociale ou en cours d'acquisition, indépendamment de la nationalité et du pays de résidence». Cruz, Armando (2004), "Portability of Benefit Rights in Response to External and Internal Labour Mobility: The Philippine Experience", paper presented at the International Social Security Association $13^{\text {th }}$ Regional Conference for Asia and the Pacific, Kuwait (8-10 March 2004).

${ }^{13}$ Sabates-Wheeler et Koettls, 2010, p. 128.

${ }^{14}$ Salin, 2014, p. 228.

${ }^{15}$ Salin, 2014, p. 210 et p. 229.

${ }^{16}$ AISS, 2014.
}

e-Revista Internacional de la Protección Social, ISNN 2445-3269. 2017, Vol. II, No 2

http://dx.doi.org/10.12795/e-RIPS.2017.i02.04. 
migrant doit donc contribuer à la protection sociale nationale sans pouvoir déterminer la frontière entre ses droits et les restrictions auxquelles il doit faire face.

L'étude de SABATES-WHEELER et KOETTLS confirme qu'une certaine disparité persiste au niveau national, entre les droits des travailleurs nationaux et ceux des travailleurs de pays tiers. En particulier, ces derniers risquent de bénéficier d'un accès restreint aux programmes de protection sociale dans les pays d'accueil en même temps qu'ils risquent de perdre les droits acquis lorsqu'ils quittent le pays tiers et de subir des pertes financières ${ }^{17}$. A ce propos, l'AISS a mis l'accent sur différents obstacles juridiques et pratiques qui affectent l'extension de la couverture aux travailleurs migrants, à partir de la croissante fragmentation du droit. Parmi les obstacles identifiés, une difficulté réelle persiste à l'affiliation au régime national de sécurité sociale du pays d'accueil, par exemple si une période minimale de résidence et de cotisations est requise pour bénéficier de la pension ou d'autres prestations sociales ${ }^{18}$.

Concernant la deuxième composante, quand on parle de portabilité, il est important de rappeler la distinction qui existe entre la transférabilité et l'exportabilité des droits aux prestations sociales ${ }^{19}$. D'une part, la transférabilité repose sur et dépend de, l'adoption de mesures de coordination entre les pays d'origine et d'accueil, tant pour la reconnaissance des droits sociaux que pour sanctionner leur violation. Il s'agit par exemple, dans le cas de prestations de pension de vieillesse, de la coordination pour la prise en compte des cotisations payées dans plusieurs pays. D'autre part, l'exportabilité n'implique pas quant à elle, une telle coordination et elle peut être réalisée par un seul pays. Tout récemment, le Représentant spécial du Secrétaire général des Nations Unies pour les migrations, M. PETER SUTHERLAND, dans son dernier rapport, a identifié parmi les priorités clés des Etats membres et des acteurs non-gouvernementaux en vue de la prochaine adoption du pacte mondial pour des migrations sûres, ordonnées et régulières, celle de «garantir l'accès aux prestations sociales acquises et leur portabilité» ${ }^{20}$. En particulier, le Représentant spécial du Secrétaire général a décrit la portabilité comme «une question d'équité pour les migrants et les pays d'origine, et une question d'efficacité de la politique migratoire pour le pays de destination. Les migrants ne doivent pas être dissuadés de retourner dans leur pays d'origine ou obligés de rester dans leur pays d'accueil plus longtemps qu'ils ne le souhaitent au simple motif qu'ils ne pourraient plus continuer à bénéficier des prestations de sécurité sociale après leur départ» ${ }^{21}$.

Comment assurer la portabilité de droits sociaux acquis ou en cours d'acquisition? La question impose la mise en relation de deux concepts: le principe de la clause de territorialité, qui autorise chaque Etat à établir certaines restrictions pour les noncitoyens, et l'affirmation du principe d'égalité démocratique garanti dans le contexte de la législation constitutionnelle nationale ${ }^{22}$. Dans une perspective historique,

\footnotetext{
${ }^{17}$ Sabates-Wheeler et Koettls, 2010, p. 125.

${ }^{18}$ AISS, 2014.

${ }^{19}$ Sabates-Wheeler et Koettls, 2010, p. 130.

${ }^{20}$ Assemblée Générale, Rapport du Représentant spécial du Secrétaire général pour les migrations, A/71/728, 2017 (http://reliefweb.int/sites/reliefweb.int/files/resources/N1700221.pdf), p. 25.

${ }^{21}$ Id. p. 22.

${ }^{22}$ Arellano Ortiz, Pablo (2013), Trabajadores Migrantes y Seguridad Social: Aproximación Nacional e International a los Mecanismos de Protección que Otorgan Continuidad a la Prestaciones. Revista Chilena de Derecho del Trabajo y de la Seguridad Social, 3(6), pp. 119-133.
}

e-Revista Internacional de la Protección Social, ISNN 2445-3269. 2017, Vol. II, No 2

http://dx.doi.org/10.12795/e-RIPS.2017.i02.04.

Página 23 
l'introduction de la clause territoriale est ancienne et elle se retrouvait déjà par exemple, dans l'évolution de la formulation de lois concernant la pauvreté et la mendicité au XVIe siècle ${ }^{23}$. Comme souligné par Brett, les lois adoptées visaient à garantir l'accès au «bien commun interne» aux personnes en détresse, sans aucune distinction entre citoyens et non-citoyens ${ }^{24}$. Leur formulation a ensuite progressivement exclu les étrangers qui n'étaient pas capables de subvenir à leur besoins ${ }^{25}$. Roberts souligne qu'au niveau territorial le traitement réservé à tout individu par le pouvoir souverain est réglé par différents instruments : les lois sur l'immigration, qui garantissent surtout l'accès au territoire, et des critères spécifiques, tels que la nationalité ou la résidence, pour assurer l'accès aux prestations.

\section{EVOLUTION HISTORIQUE DU CADRE NORMATIF INTERNATIONAL}

Sur le plan historique, la protection des droits sociaux a été incluse dans le droit international des droits de l'homme à partir de l'adoption de la Déclaration universelle de droits de l'homme en 1948. Cette dernière reconnaît à l'article 22, «le droit à la sécurité sociale» et précise à l'article 25 , «le droit à la sécurité en cas de chômage, de maladie, d'invalidité, de veuvage, de vieillesse ou dans tous les autres cas de perte de ses moyens de subsistance par suite de circonstances indépendantes de sa volonté». Les dispositions de la Déclaration sont traduites par les deux Pactes contraignants adoptés par les Nations Unies en 1966. En particulier, l'article 2 du Pacte sur les droits civils et politiques qui interdit toute forme de discrimination et l'article 9 du Pacte des droits économiques, sociaux et culturels qui prévoit «le droit de toute personne a la sécurité sociale, y compris les assurances sociales». Le respect de ces droits implique donc qu'il est inacceptable pour l'ensemble des pays de les limiter et d'exclure les travailleurs migrants de leur jouissance.

Dans ce contexte, l'Organisation internationale du travail (OIT) a sûrement joué un rôle clé dans l'évolution du cadre normatif de la protection sociale des travailleurs migrants. Il est d'abord souligné comment la question liée à la portabilité des pensions a évolué au sein de l'organisation. En effet, il est surprenant de voir que la question avait déjà été abordée au sein de la Conférence internationale du Travail en 1927 quand M. Martens (à l'époque délégué ouvrier belge) a soumis un projet de résolution concernant l'assurance en cas d'invalidité, de vieillesse et de décès, et la conservation des droits de pension pour les travailleurs migrants ${ }^{26}$. Lors des débats sur cette résolution, les Etats membres ont demandé au Conseil d'administration du Bureau international du travail d'aborder la question «de la conservation des droits à pension des travailleurs qui passent d'un pays dans un autre» ${ }^{27}$.

Cette question a été mise à l'ordre du jour lors de la Conférence de 1933 et a conduit à la mise en chantier de la première Convention pour la conservation des droits et à l'établissement d' «un véritable régime international pour la protection des salariés

\footnotetext{
${ }^{23}$ Brett, S. Annabel (2011), Changes of State, Nature and the limits of the city in the early modern natural law, Princeton University Press.

${ }^{24}$ Brett, 2011.

${ }^{25}$ Roberts, 2010.

${ }^{26}$ Conférence Internationale du Travail (CIL, 1927), Compte Rendu des Travaux, Dixième Session, vol. I, p. 529.

${ }^{27} \mathrm{CIL}, 1927$, p. 532 ; Pour le texte de la résolution voir CIL, 1927, pp. 680-682.
}

e-Revista Internacional de la Protección Social, ISNN 2445-3269. 2017, Vol. II, No 2

http://dx.doi.org/10.12795/e-RIPS.2017.i02.04.

Página 24 
travaillant dans plusieurs pays» ${ }^{28}$. La Commission de la conservation des droits à pension (par la suite la Commission) a œuvré dans le but d'élaborer ce texte pour aboutir «à poser la première pierre d'une Union internationale pour la protection d'assurance des travailleurs de tous les pays» ${ }^{29}$. La Commission a présenté la Convention $\left(\mathrm{n}^{\circ} 48\right)$ sur la conservation des droits à pension des migrants, qui a été adoptée en $1935^{30}$. Dans cette Convention, le principe de la conservation des droits acquis et en cours d'acquisition a été au centre des discussions et des débats qui ont conduit à l'adoption du texte final sur la base de la conviction que «chacun doit recevoir les prestations qui correspondent à l'effort qu'il a fourni» ${ }^{31}$. Cette Convention n'a pas reçu un nombre significatif de ratifications. C'est l'une des raisons qui a amené la Commission internationale du Travail à la réviser en $1981^{32}$ notamment du fait de sa «rigidité» et de sa limitation à trois régimes «les prestations de vieillesse, d'invalidité et de survivants» ${ }^{33}$. Ceci a, par la suite, déterminé l'adoption d'une nouvelle Convention $\left(n^{\circ} 157\right)$ en $1982^{34}$. Comme énoncé par la CIT «contrairement à la convention $n^{\circ} 48$, la nouvelle convention qui concerne toutes les branches ainsi que l'ensemble des prestations et régimes de sécurité sociale, contributifs et non contributifs ne comporte de dispositions directement applicables même en l'absence d'instruments bilatéraux ou multilatéraux entre les Etats liés par elle, que sur un nombre limité de points faisant l'objet des dispositions visées au paragraphe 2 de son article $4 »^{35}$. En particulier, la situation historique était différente de celle de l'adoption de la Convention $n^{\circ} 48$, étant donné que la période de l'après-guerre a vu une croissance significative de la coordination dans le contexte de la protection sociale à travers une multiplication des accords bilatéraux. Ceci a favorisé une prise de conscience du besoin de développer un instrument multilatéral pour améliorer la coordination entre ces instruments ${ }^{36}$.

\footnotetext{
${ }^{28}$ CIL, 1934, p. 436.

${ }^{29}$ Lors de séances de travail de la Commission pour la conservation des droits à pension, les Etats membres ont, à plusieurs reprises, souligné l'importance dans ce domaine des accords bilatéraux conclus par exemple par l'Espagne (CIL, 1934, p. 436, M. Stern, conseiller technique gouvernemental, Tchécoslovaquie).

${ }^{30} \mathrm{La}$ Convention $\mathrm{n}^{\circ} 48$ a été uniquement ratifiée par un nombre non significatif de pays : Italie (1952), Israël (1963), Monténégro (2006), Serbie (2000), Slovénie (1992), Croatie (1991) et Bosnie-Herzégovine (1993). Voir pour le résumé de travaux préparatoires : Conférence Internationale du Travail (CIL, 1935a), Rapport de la Commission de la conservation des droits à pension : discussion, p. 553 ; Conférence Internationale du Travail (CIL, 1935b), Annexe VI : Première question à l'ordre du jour : Conservation au profit des travailleurs qui transfèrent leur résidence d'un pays à un autre, des droits en cours d'acquisition et des droits acquis dans l'assurance invalidité-vieillesse-décès, p. 771.

${ }^{31}$ CIL, 1935 a, p. 554.

${ }^{32}$ Conférence Internationale du Travail (CIL, 1981), Compte rendu provisoire 32, Soixante-septième session, p. 3: «le petit nombre de ratifications reçues par cette convention, en dépit de la source indéniable d'inspiration qu'elle a constituée sur le plan mondial pour de nombreuses conventions bilatérales et multilatérales de sécurité sociale. Les raisons de ce relatif insuccès tiennent sans doute au caractère trop ambitieux et trop rigide de cette convention, qui établit un système international de conservation des droits directement applicable, sans que l'on ait pu prévoir le très grand développement ultérieur des instruments bilatéraux et multilatéraux de sécurité sociale qu'elle a directement influencés ». ${ }^{33}$ CIL, 1981, pp. 32-33 ; Conférence Internationale du Travail (1982), Compte rendu provisoire 33, Soixante-huitième session, p. $6:$ «e membre gouvernemental des Etats-Unis, notant que la commission traite de la conservation des droits des «travailleurs migrants » en matière de " sécurité sociale », a estimé que l'aspect sécurité sociale était primordial, car ce sont toutes les catégories de personnes protégées appelées à se déplacer d'un pays à un autre qui pourraient bénéficier de la convention ».

${ }^{34}$ Conférence Internationale du Travail (CIT, 1983), Conservation des droits en matière de sécurité sociale, Rapport V, Soixante-neuvième session.

${ }^{35}$ CIT, 1983 , p. 5.

${ }^{36} \mathrm{CIT}, 1983$, pp. 5-6 : «Enfin, les instruments de coordination, lorsqu'ils sont de nature bilatérale, comme c'est le cas de la majorité d'entre eux, n'apportent que des solutions imparfaites dans toutes les situations
}

e-Revista Internacional de la Protección Social, ISNN 2445-3269. 2017, Vol. II, No 2

http://dx.doi.org/10.12795/e-RIPS.2017.i02.04.

Página 25 
En même temps, l'OIT a adopté des instruments très importants dans ce domaine à partir de conventions relatives à la non-discrimination et à l'égalité des chances pour les migrants dans le pays d'accueil ${ }^{37}$. En ce qui concerne l'application de ces textes, elle est soumise à la condition de réciprocité, c'est-à-dire que les dispositions contenues dans ces textes ne sont valables que pour les ressortissants des Etats parties à ces conventions. Le champ d'application est plus étendu pour la Convention $n^{\circ} 97$ sur les travailleurs migrants de 1949. Cette dernière est applicable sans condition de réciprocité ; l'art. 6.1, en particulier, affirme l'égalité de traitement pour l'accès à la sécurité sociale ${ }^{38}$. Plus récemment, en 2012, l'OIT a adopté la recommandation $n^{\circ} 202$ relative aux socles nationaux de protection sociale pour garantir la couverture de tout résident et pas uniquement des citoyens ${ }^{39}$.

Face à l'insuffisance des dispositions adoptées au niveau national, on assiste à une coordination progressive, soit au niveau bilatéral, soit au niveau régional, qui contribue à concrétiser le principe d'égalité garanti en droit international et à permettre aux citoyens qui se trouvent hors de leur pays d'origine de bénéficier de prestations sociales ${ }^{40}$.

Les deux sections suivantes visent à explorer deux instruments différents de coordination adoptés par les Etats et leur impact vis-à-vis de la protection des migrants : les conventions bilatérales de sécurité sociale et les régimes régionaux.

\section{LES ACCORDS BILATERAUX}

Si le thème de la protection sociale se situe dans un contexte institutionnel en apparence dominé par une conception classique de la souveraineté nationale, des transformations nouvelles du rôle de l'Etat, associées aux modes de la gouvernance de la migration, garantissent la progression de l'accès aux droits sociaux sur le territoire national. En effet, il convient de souligner que la gouvernance de la migration implique des formes différentes de l'action étatique. Il est question d'un processus visant à favoriser une «transnationalisation» croissante de la gouvernance de la migration avec un accent de plus en plus important sur des modes bilatéraux et régionaux de formulation de politiques migratoires.

où les personnes intéressées sont ressortissantes d'un Etat tiers, ainsi que dans celles où elles ont également commencé à acquérir des droits au titre de la législation d'un Etat tiers ou bien résident ou séjournent sur le territoire d'un tel Etat, situations de plus en plus fréquentes compte tenu de la complexité des mouvements migratoires. A défaut d'instruments multilatéraux qui se substituent à des instruments bilatéraux ou en dispensent dans les relations entre les Etats en cause, il importe de faciliter et d'encourager la coordination multilatérale des instruments bilatéraux existants ».

37 Convention ( $\left.{ }^{\circ} 19\right)$ sur l'égalité de traitement (accidents du travail) de 1925 (qui, au 30 novembre 2015 , comptait 121 ratifications) et recommandation $n^{\circ} 25$; Convention $\left(n^{\circ} 21\right)$ relative à l'inspection des émigrants de 1926 ; Recommandation ( $\mathrm{n}^{\circ}$ 26) sur la migration (protection des émigrantes abord des navires) de 1926 ; Convention ( $\left.\mathrm{n}^{\circ} 48\right)$ sur la conservation des droits à pension des migrants de 1935.

${ }^{38}$ Gisti (2016), La Protection Sociale des personnes étrangères par les textes internationaux.

${ }^{39}$ AISS, 2014.

${ }^{40}$ Gisti, 2016.

e-Revista Internacional de la Protección Social, ISNN 2445-3269. 2017, Vol. II, Nº 2

http://dx.doi.org/10.12795/e-RIPS.2017.i02.04. 
Un certain nombre de pays ont conclu des accords bilatéraux relatifs à la sécurité sociale pour garantir la protection sociale des travailleurs migrants ${ }^{41}$. Dans cette optique, les instruments adoptés peuvent avoir comme objectif l'harmonisation ou la coordination des régimes nationaux ${ }^{42}$. MAHON, dans son étude, rappelle clairement la distinction entre l'harmonisation «ayant pour but de favoriser le rapprochement matériel des différents systèmes nationaux» et la coordination qui vise non pas à modifier les différents systèmes nationaux mais à permettre aux migrants «de passer de l'un à l'autre sans subir les inconvénients liés à cette mobilité et au caractère territorial des systèmes nationaux» ${ }^{43}$. Comme souligné par Watson, «la coordination relie les systèmes juridiques : elle n'altère pas leur substance sauf dans la mesure où elle accroît la portée des systèmes de sécurité sociale en rendant les droits personnels plutôt que territoriaux ${ }^{44}$.Très peu des pays concernés visent à développer une harmonisation dans ce domaine ${ }^{45}$, et la plupart ont développé une pratique de coordination.

Il est particulièrement intéressant de rappeler l'accord bilatéral de coordination en matière de sécurité sociale adopté entre la France et l'Italie en $1904^{46}$. Comme énoncé dans la première partie de la Convention, le traité visait à : «1 $1^{\circ}$ faciliter à leurs nationaux travaillant à l'étranger la jouissance de le leurs épargnes et leur ménager le bénéfice des assurances sociales $; 2^{\circ}$ garantir aux travailleurs le maintien des mesures de protection déjà édictées en leur faveur et concourir au progrès de la législation ouvrière». L'adoption de règles internationales a été invoquée depuis de nombreuses années dans différents fora internationaux, comme la Conférence de Berlin (1890), le Congrès de Zurich (1897), ainsi que le Congrès de Paris (1900) qui a facilité que les négociations officielles commencent en $1904^{47}$. Cet accord bilatéral incluait le principe de la réciprocité de traitement entre les citoyens de deux pays et il garantissait le transfert d'épargne (article 1) et la facilitation du transfert de pensions et cotisations

\footnotetext{
${ }^{41}$ Conférence Internationale du Travail (CIT, 2011), La sécurité sociale pour la justice sociale et une mondialisation équitable, Discussion récurrente sur la protection sociale (sécurité sociale) en vertu de la Déclaration de l'OIT sur la justice sociale pour une mondialisation équitable, Genève, Bureau International du Travail, p. 35.

${ }^{42}$ Dupper, Ockert (2014), "Coordination of Social Security Schemes, the Case of SADC", KFG The transformative power of Europe, Working Paper, n. 60, p. 16; Pennings, Frans (2012), EU Citizenship : Access to Social Benefits in Other EU Member States, The International Journal of Comparative Labour Law and Industrial Relations, 28 (3), 307-334.

${ }^{43}$ Mahon, Pascal (1993), «Etrangers, travailleurs migrants et sécurité sociale », Aspects de la sécurité sociale, vol. 2, n. 3, Genève, pp. 3-9, 1-12, p. 5 et 1.

${ }^{44}$ Watson, Philippa (1980), Social Security Law of the European Communities, Mansell Publishing, p. VIII: "coordination links legal systems: it does not alter their substance except in so far as it widens the scope of social security systems by rendering rights personal rather than territorial".

${ }^{45}$ Voir art. 2 (e) de la Charte des droits sociaux de la Communauté de développement d'Afrique australe (SADC) : " the establishment and harmonisation of social securityschemes".

${ }^{46}$ Le texte de l'accord a été publié par le Bollettinodell'UfficiodelLavoro, Vol. I, n. 3, Juin 1904, pp. 317 335. En France, le décret du 8 octobre concernant la Convention du travail franco-italien du 15 avril 1904 a été publié par le Bulletin de l'inspection du travail, deuxième année, 1904, pp. 325-331. La Convention a été signée à Rome le 15 avril 1904 avec un arrangement pour régler les remboursements et les transferts de fonds entre les deux pays. Les échanges de ratification ont eu lieu le 21 septembre 1904. Pour une analyse détaillée de ce texte voir en particulier : Pic, Paul (1906), De la condition juridique des travailleurs étrangers en France, Législation actuelle, Projets de réforme, Journal du droit international privé, pp. 301-317; Raynaud, B. (1905), Le Traité de travail franco-italien du 15 avril 1904, Journal du droit international privé, pp. 306-316.
}

${ }^{47}$ Raynaud, 1905, p. 307.

e-Revista Internacional de la Protección Social, ISNN 2445-3269. 2017, Vol. II, No 2

http://dx.doi.org/10.12795/e-RIPS.2017.i02.04. 
(article $1, b)^{48}$ qui ne pouvait pas se faire dans l'immédiat en raison des différences de degré dans l'évolution législative nationale des deux pays ${ }^{49}$. De plus, il affirme l'égalité de traitement entre les citoyens de deux pays dans l'accès aux indemnités en cas d'accident s'ils quittent le territoire où l'accident a eu lieu (article 1, d). Ce traité a contribué à identifier les éléments de base du principe de la coordination en matière de protection sociale et à mettre l'accent sur la condition des travailleurs migrants. ${ }^{50} \mathrm{Il}$ a été souligné que cet accord en a inspiré d'autres, comme le traité commercial conclu entre l'Italie et la Suisse le 13 juillet $1904^{51}$. En particulier, selon HOLLOWAY, l'adoption des premiers accords internationaux a eu pour rôle d'adresser le «paradigme du libéralisme-protectionnisme» qui intéressait les Etats à cette période dans la gestion de leurs flux migratoires. En effet, ces derniers faisaient face, d'un côté, à la nécessité de contrôler l'entrée et le séjour des migrants et, de l'autre côté, à la nécessité de garantir un nombre donné de travailleurs migrants dans certains Etats. L'évolution des lois migratoires internes a donc été accompagnée, en même temps, par ces accords internationaux aptes à favoriser la libre circulation des travailleurs migrants entre les Etats parties. L'accord franco-italien a alors été identifié comme une possible solution aux tensions liées au dilemme du libéralisme-protectionnisme ${ }^{52}$.

A l'heure actuelle, en matière de coordination de la sécurité sociale, les conventions bilatérales jouent un rôle clé dans la sauvegarde des droits à la protection sociale pour les personnes en situation de mobilité. Elles constituent notamment un instrument important de coordination en matière de sécurité sociale pour les Etats qui ne sont parties à aucun accord multilatéral ou supranational ${ }^{53}$. En tant qu'instrument de coordination, les conventions bilatérales visent à améliorer la protection de travailleurs migrants «en créant des «ponts» entre les différents systèmes, de façon à faciliter le passage de l'un à l'autre, sans toutefois modifier ces systèmes»» ${ }^{54}$. Un objectif important lié à l'adoption de ces instruments est la concrétisation du principe d'égalité de traitement entre les étrangers migrants et les ressortissants du pays d'accueil dans l'accès aux prestations de sécurité sociale ${ }^{55}$. Comme souligné par l'OIT, «la multiplication des accord bilatéraux et multilatéraux de sécurité sociale» garantit «l'application dans la pratique du principe de l'égalité de traitement grâce à des dispositions qui permettent le maintien des droits acquis et des droits en cours d'acquisition ${ }^{56}$.

\footnotetext{
${ }^{48}$ Watson, 1980, p. 9. Il est intéressant de souligner que l'accord concernait 200.000 citoyens italiens (employés en France) et 10000 citoyens français (employés en Italie). Si l'avantage pour l'Italie était assez évident, pour la France l'intérêt résidait plutôt dans la deuxième partie de l'accord qui imposait à l'Italie d'améliorer sa législation interne en matière de protection sociale. En effet, il était estimé que les coûts de production soutenus par les employeurs en Italie étaient très bas par rapport à la France, en raison d'un cadre législatif inadéquat et sous-développé, Holloway, John (1981), Social Policy Harmonisation in the European Community, Gower, p. 251.

${ }^{49}$ En particulier, l'Italie avait adopté deux lois, en 1898 et en 1901, pour reconnaître le droit aux pensions pour les travailleurs mais la France n'avait pas encore avancé dans ce domaine, voir Watson, 1980, p. 9.

${ }^{50}$ Watson, 1980, p. 9 ; Holloway, 1981 , p. 251.

${ }^{51}$ Le texte de cet accord a été publié dans la Feuille Fédérale, décembre 1904, vol. 6, n. 49, pp. 148 - 239.

${ }^{52}$ Holloway, 1981, pp. 243-245.

${ }^{53}$ MISSOC (Mutual Information System on Social Protection in the EU Member States, the EAA and Switzerland), Analyse MISSOC, 2/2013, Aspects extérieures de la coordination en matière de sécurité sociale, Novembre 2013, p. 17.

${ }^{54}$ Mahon, 1993, p. 5.

${ }^{55} \mathrm{Gisti}, 2016$.

${ }^{56}$ OIT, 2016.
}

e-Revista Internacional de la Protección Social, ISNN 2445-3269. 2017, Vol. II, No 2

http://dx.doi.org/10.12795/e-RIPS.2017.i02.04. 
Les raisons sous-tendant les conclusions de ces accords sont très variées. Historiquement, l'adoption de ces accords était liée à la «naissance des assurances sociales» ${ }^{57}$ pour évoluer, au fil des années, avec le développement des politiques migratoires. Si on considère, par exemple, le cas de la France qui, à l'heure actuelle, a conclu environ 38 accords bilatéraux avec des pays tiers ${ }^{58}$, on peut clairement distinguer 3 séries d'accords : 1 - les accords dits de «main-d'œuvre » conclus à partir des années 1950, lors de la décolonisation, avec les pays du Maghreb et d'Afrique pour favoriser la mobilité des travailleurs migrants de ces pays ; 2 -les accords de mobilité conclus dans les années 1980 avec de nouveaux Etats d'Amérique et d'Asie ; 3- les accords dits «OCDE» signés à partir des années 2000 pour les échanges avec les pays industrialisés ${ }^{59}$. Aussi, l'ensemble des accords bilatéraux signés par les pays du Mercosur (Marché commun du Sud) avec d'autres pays d'Amérique Latine, en particulier avec la Colombie et le Chili, sont étroitement liés à une logique de mobilité des travailleurs comme le démontre l'étude développée par BERNAL, PRADA et URUEÑ $^{60}$. A partir du milieu des années 1990, une nouvelle série d'accords ont été signés avec les pays d'Amérique latine dans une logique de migration Sud-Sud. ${ }^{61}$ Depuis les années 2000, le réseau des pays d'Amérique du Sud s'est étendu à l'Espagne en raison de l'accroissement des échanges de flux migratoires avec ce pays.

En ce qui concerne le champ d'application personnel, à savoir les personnes couvertes, on distingue entre des accords «fermés» qui sont valables seulement pour les ressortissants des parties contractantes ${ }^{62}$ et des accords «ouverts» qui s'appliquent à toutes personnes couvertes par la législation en matière de sécurité sociale, quelle que soit leur nationalité6 ${ }^{63}$. Il est difficile d'identifier la prévalence d'un modèle. En effet, certains accords plus récents sont «fermés» donc restreints aux ressortissants nationaux ${ }^{64}$. Même si ces accords sont très hétérogènes, ils partagent des caractéristiques communes en ce qui concerne le champ d'application matériel, ${ }^{65}$ à savoir les éventualités. Ces accords visent à promouvoir l'égalité de traitement entre les ressortissants des deux Etats signataires, ainsi que le maintien des droits acquis et en cours d'acquisition ${ }^{66}$. Dans cette optique, ces accords garantissent la possibilité d'exporter certaines prestations, telles que les pensions de vieillesse, d'invalidité, de survivants et pour les prestations dans le cadre d'accidents du travail et de maladies professionnelles. Selon l'analyse du MISSOC, plusieurs accords conclus par les pays européens couvrent les branches classiques de la sécurité sociale et la plupart des

\footnotetext{
${ }^{57}$ Mahon, 1993, p. 1.

${ }^{58}$ Gisti, 2016.

${ }^{59}$ Réseau européen des migrations (REM) (2014), L'accès des ressortissants de pays tiers à la sécurité sociale et aux soins de santé en France, Février 2014, p. 35.

${ }^{60}$ Voir par exemple les accords conclus entre l'Argentine et la Colombie en 2008, entre le Brésil et le Chili en 1995, et entre l'Uruguay et le Mexique en 1990. Bernal, Natalia, Prada, María et Urueña, René (2015), "Intra-regional Mobility in South America: the Andean Community and MERCOSUR", dansPanizzon, Marion, Zuercher, Gottfried et Fornalé, Elisa (dir.), The Palgrave Handbook of International Labour Mobility, London, Palgrave.

${ }^{61}$ Voir par exemple les accords conclus entre le Brésil et le Portugal en 1991. Bernal, Prada et Urueña, 2015.

62 Par exemple, l'analyse MISSOC 2/2013 énumère les accords conclus par Malte et la Roumanie.

${ }^{63}$ Voir les accords bilatéraux conclus par le Chili. MISSOC, 2013, p. 18.

${ }^{64}$ Par exemple, l'accord conclu entre la France et le Kosovo (2013) ou l'accord conclu entre la Roumanie et le Canada (2011). MISSOC, 2013, p. 26.

${ }^{65}$ MISSOC, 2013, p. 17.

${ }^{66}$ Arellano Ortiz, 2013.
}

e-Revista Internacional de la Protección Social, ISNN 2445-3269. 2017, Vol. II, No 2

http://dx.doi.org/10.12795/e-RIPS.2017.i02.04. 
accords n'incluent pas la portabilité de l'indemnité de chômage et des allocations familiales ${ }^{67}$. Cette distinction entre les transferts et les non-transferts assurés par les Etats aux étrangers fait apparaître le caractère arbitraire de la cotisation et engendre une «inégalité» spécifique ${ }^{68}$.

Sur la base des études disponibles, il apparaît que la coordination réalisée à travers les accords bilatéraux de sécurité sociale est disparate. Ceci laisse la place à des lacunes conséquentes et concrètes au niveau de la réalisation du principe d'égalité. En particulier, certaines restrictions dans le traitement réservé aux étrangers peuvent affecter l'assujettissement à un régime, sur la base du critère de la nationalité, ou le droit et les conditions d'octroi des prestations qui affectent la réalisation d'une égalité globale. De plus, il convient de mentionner que plusieurs pays d'origine n'ont conclu aucun accord bilatéral de transférabilité. Les migrants dépendent donc uniquement de la législation nationale qui, dans certains pays, n'est pas suffisante. Par conséquent, comme récemment développé dans l'étude publiée par l'OIT concernant l'accès à la protection sociale dans les pays d'Asie du Sud, les travailleurs migrants encourent le risque d'une double limitation : soit de ne pas avoir accès aux prestations et aux droits associés aux cotisations obligatoires dans leur pays d'origine, soit d'en perdre l'accès une fois qu'ils retournent dans leur pays d'origine ${ }^{69}$.

L'origine historique de cette exclusion confirme la volonté des Etats de faire prévaloir leur prérogative souveraine sous prétexte qu'il ne serait pas possible d'effectuer de contrôle hors des frontières nationales ni de vérifier que les bénéficiaires remplissent les conditions pour le maintien de leur droit aux prestations (voir par exemple l'aptitude au placement d'un chômeur ${ }^{70}$. Il devient de plus en plus urgent de se demander si cette contrainte imposée par l'Etat est «admissible» et si elle répond à celle que l'on appelle la «justice sociale» ou la «solidarité obligatoire», selon la définition adoptée par SALIN $^{71}$. En effet, la solidarité étatique, d'après la formule de Salin, peut dégénérer en une spoliation légale au détriment des travailleurs migrants si elle franchit la limite du droit. Le travailleur migrant ne peut pas invoquer une dénationalisation de l'assurance chômage étant donné qu'il paie lui-même ses cotisations et qu'il perd ces dernières s'il quitte le pays d'accueil.

En règle générale, «les travailleurs migrants contribuent aux systèmes de sécurité sociale le plus souvent sans que leurs droits soient garantis. La portabilité et le maintien des droits acquis doivent être considérés comme des droits de base ${ }^{72}$.

\footnotetext{
${ }^{67}$ Par exemple l'accord conclu entre la Belgique et le Maroc (1971) ou l'accord conclu entre la Bulgarie et la Russie (2010).

${ }^{68}$ Holzmann dans son étude a aussi mis en évidence comment la neutralité de ces accords peut être touchée par la mise en place d'un traitement fiscal diffèrent lors de la portabilité de pensions, qui peut varier selon les pays concernés. Holzmann, 2016, p. 119 et p. 123.

${ }^{69}$ OIT (2015), The State of Social Protection in ASEAN at the dawn of integration, OIT Bangkok.

${ }^{70}$ Mahon, 1993, p. 7.

${ }^{71}$ Salin, 2014, p. 262.

${ }^{72}$ Processus de Rabat (Soutien au processus Euro-Africain sur la Migration et le Développement 2011), «Rapport Final», Réunion d'experts sur les droits sociaux des migrants et leur portabilité dans un cadre international, Rabat, [en ligne], http://www.processusderabat.net/web/uploads/activity/Social_rights/S\%C3\%A9ance\%207_ILO_FR.pdf, p. 6.
}

e-Revista Internacional de la Protección Social, ISNN 2445-3269. 2017, Vol. II, No 2 


\section{L'EVOLUTION DE LA COORDINATION DE LA SECURITE SOCIALE AU NIVEAU REGIONAL}

Si certains pays ont démontré une attitude positive envers l'adoption des conventions bilatérales, il existe également des instruments de sécurité sociale visant à promouvoir une coordination multilatérale qui pourrait avoir un impact très important à l'égard des étrangers et à la reconnaissance du principe d'égalité. En particulier, le risque lié aux conventions bilatérales est de favoriser une attitude sélective et restrictive des pays concernés. Au contraire, une ouverture sur le multilatéralisme pourrait renforcer l'application des normes internationales.

Au niveau régional, l'Union européenne (UE) a développé un système de règles qui vise à coordonner les systèmes de sécurité sociale pour favoriser la libre circulation des personnes $^{73}$, et ce depuis plus de 50 ans $^{74}$. Le système mis en place figure parmi le plus développé $^{75}$ au niveau régional avec la concrétisation du principe de coordination interétatique. L'évolution du cadre normatif européen en matière de droits sociaux a été strictement liée à la mise en place du principe de la libre circulation de travailleurs ${ }^{76}$. L'introduction de l'article $51 \mathrm{du}$ Traité de Rome a favorisé une «déterritorialisation» progressive de la protection sociale pour garantir la libre circulation ${ }^{77}$. Parmi les principes fondamentaux qui visent à garantir l'accès à la protection sociale, on retrouve: le principe de non-discrimination en tant que «principe fondamental, directement applicable, obligatoire pour les Etats membres», et la portabilité des prestations sociales et le regroupement des périodes de prestations ${ }^{78}$. Le respect de ce dernier principe est en outre garanti par la Cour de Justice de la Communauté européenne avec une attention particulière au champ d'application matériel. D'autre part, le régime européen a progressivement évolué pour inclure les ressortissants de pays tiers dans le champ d'application des règles communautaires ${ }^{79}$. A l'heure actuelle, il inclut différents instruments: plusieurs directives adoptées pour des catégories spécifiques de travailleurs migrants ${ }^{80}$.

\footnotetext{
${ }^{73}$ More, Gillian (2011), " Règles de coordination de la sécurité sociale de l'union européenne développement d'une dimension externe, Présentation à la réunion d'experts sur les droits sociaux des migrants et leur portabilité dans un cadre transnational », Rabat, Maroc 31 mars - 1 avril 2011, p. 2.

${ }^{74}$ Voir les règles énoncées dans le règlement $(\mathrm{CE}) \mathrm{n}^{\circ} 883 / 2004$ portant sur la coordination des systèmes de sécurité sociale et le règlement d'application (CE) n 987/2009 (JO L 166 du 30.5.2004, p. 1 et JO L 284 du 30.10.2009, p. 1). Les principes de base de la coordination en matière de sécurité sociale incluent : l'égalité de traitement indépendamment de la nationalité, le cumul des périodes d'assurances acquises sous les différents systèmes nationaux, la portabilité des droits sociaux à l'intérieur du territoire de l'UE, une seule législation applicable et la coopération administrative entre les différents institutions de sécurité sociale (Dupper, 2014, p.17). VoiraussiPennings, 2012 ; Sánchez-Rodas Navarro, Cristina (Ed.) (2010), Migrants and Social Security, The EC Regulations 883/2004 and 987/2009 (Madrid: EdicionesLaborum).

${ }^{75}$ Mahon, 1993, p. 7.

${ }^{76}$ Sánchez-Rodas Navarro, 2010.

${ }^{77}$ Watson, 1980, p. 35.

${ }^{78}$ Avec l'adoption du règlement (CE) 1231/10, la catégorie de travailleurs migrants se trouvant en situation de transfrontaliers est également concernée par les règles de coordination. Pour l'application de ce règlement deux conditions sont requises : 1 - avoir une résidence légale dans un Etat Membre et 2 être «dans une situation non-confinée en tous points à un seul Etat membre ». Mahon, 1993, pp. 3-4 ; AISS, 2014.

${ }^{79}$ Voir le «Programme d'action en faveur des travailleurs migrants et de leurs familles » adopté en 1974 pour favoriser la reconnaissance de même traitement aux travailleurs migrants de pays tiers, en particulier pour souligner l'importance de reconnaître la portabilité de droits sociaux.

${ }^{80}$ Voir respectivement: la Directive 2009/50/CE du Conseil du 25 mai 2009 sur les conditions d'entrée et de séjour des ressortissants de pays tiers aux fins d'un emploi très qualifié, JO 2009, L 155/17 (directive
}

e-Revista Internacional de la Protección Social, ISNN 2445-3269. 2017, Vol. II, No 2

http://dx.doi.org/10.12795/e-RIPS.2017.i02.04.

Página 31 
La Commission européenne a lancé, en 2012, une Communication sur la dimension extérieure des règles de coordination de la sécurité sociale dans l'Union Européenne pour assurer le développement d'une stratégie commune vis-à-vis des pays tiers ${ }^{81}$. En particulier, l'UE envisage d'adopter des nouveaux accords pouvant introduire un modèle novateur de protection des travailleurs migrants en matière de sécurité sociale. Elle vise à encourager le développement d'une approche globale en ce qui concerne les prestations de sécurité sociale aux citoyens de l'UE et aux ressortissants de pays tiers.

Sur la base de l'égalité de traitement et de l'interdiction des discriminations fondées sur la nationalité, «un pays de l'UE permet à ses ressortissants, de par la loi, d'exporter leur pension à un pays tiers, le ressortissant d'un pays tiers relevant du champ des règles de coordination doit aussi avoir le droit d'exporter sa pension au pays tiers sous les mêmes conditions qu'un ressortissant de cet État membre» ${ }^{82}$. En outre, il convient de rappeler que les règles communautaires en matière de sécurité sociale priment sur les dispositions nationales établies dans des accords bilatéraux avec des Etats tiers ${ }^{83}$. En effet, les accords bilatéraux conclus par les Etats membres avec des pays tiers n'échappent pas au principe de primauté du droit de l'Union européenne.

Dans le contexte régional, il est nécessaire de mentionner d'autres accords en place qui visent à soutenir et à garantir la protection sociale des travailleurs migrants et de leurs familles. Ces accords peuvent contribuer à modifier l'approche de la garantie de la protection sociale en l'absence de protection dans les pays d'origine et d'accueil, quand bien même, comme l'a soulevé l'étude de SABATES-WHEELER et KOETTLS, un certain nombre de limitations, telles que «le manque de capacité administrative» au niveau national, persistent et peuvent affecter la couverture assurée par les pays à faible revenu ${ }^{84}$.

A titre d'exemple, la Communauté de développement d'Afrique australe (CDAA) a adopté le Code social relatif aux droits des migrants qui vise à encourager la coordination et l'harmonisation des initiatives nationales pour renforcer la protection

sur la carte bleue européenne) ; la Directive 2011/98/UE du Parlement européen et du Conseil du 13 décembre 2011 établissant une procédure de demande unique en vue de la délivrance d'un permis unique autorisant les ressortissants de pays tiers à résider et à travailler sur le territoire d'un Etat membre et établissant un socle commun de droits pour les travailleurs issus de pays tiers qui résident légalement dans un Etat membre, JO 2011, L 343/1 (directive européenne sur le permis unique) ; la Directive 2014/36/UE du Parlement européen et du Conseil du 26 février 2014 établissant les conditions d'entrée et de séjour des ressortissants de pays tiers aux fins d'un emploi en tant que travailleur saisonnier, JO 2014, L 94/375 ; la Directive 2014/66/UE du Parlement européen et du Conseil du 15 mai 2014 établissant les conditions d'entrée et de séjour des ressortissants de pays tiers dans le cadre d'un transfert temporaire intragroupe, JO 2014, L 157/1.

${ }^{81}$ COM (2012)153 final, 30 mars 2012. Il est intéressant de rappeler que l'application territoriale du Traité de Rome en dehors de l'Europe a été soulevé devant la Cour de Justice dans plusieurs cas, comme en 1970 dans l'affaire Merluzzi v. C.P.C.A.M. (case 80/71) qui concernait un citoyen italien résidant au Maroc qui souhaitait contribuer au système d'assurance sociale en France; ou encore dans l'affaire Allgemeine OrtskrankenasseHamburg v. Landesversicheringsanstalt Schleswig-Holstein (case 16/72, 1972), pour l'inclusion de contributions versées dans des pays non-membres. Voir Watson, 1980, p. 118.

${ }^{82}$ More, 2011, p. 5.

${ }^{83}$ Commission Européenne (2012), «La dimension extérieure de la coordination en matière de sécurité sociale dans l'Union européenne», Communication de la Commission au Parlement Européen, au Conseil, au Comité économique et social européen et au Comité des régions, Bruxelles, COM(2012) 0153 final, p. 4.

${ }^{84}$ Sabates-Wheeler et Koettls, 2010, p. 143.

e-Revista Internacional de la Protección Social, ISNN 2445-3269. 2017, Vol. II, Nº 2

http://dx.doi.org/10.12795/e-RIPS.2017.i02.04. 
sociale des travailleurs migrants ${ }^{85}$. Toutefois, l'absence de force contraignante de cet instrument risque d'affecter son application et la coordination elle-même entre les systèmes de sécurité des pays concernés ${ }^{86}$, notamment en ce qui concerne le cumul de périodes d'assurances, l'exportabilité et le maintien de droits acquis ${ }^{87}$. Il n'en demeure pas moins que son rôle reste très important étant donnée l'absence quasi totale d'accords bilatéraux conclus par les pays membres de ce bloc régional ${ }^{88}$.

D'autre part, il existe un accord sur la sécurité sociale adopté en 1997 par les Etats membres du Secrétariat de la Communauté des Caraïbes (CARICOM), qui vise à harmoniser la législation relative à la sécurité sociale des Etats Membres. Cet accord encourage ces derniers à octroyer une égalité de traitement dans l'accès au système de sécurité sociale et à offrir une protection pour le maintien des droits acquis ou en cours d'acquisition. Dans son analyse, FORTEZA conclut que les différences normatives qui existent au niveau national et leur méconnaissance de la part de leur destinataire affaiblissent sensiblement l'impact régional ${ }^{89}$.

Quant aux pays du Golfe, ils ont adopté en 2006, dans le cadre du Conseil de coopération du Golfe $(\mathrm{GCC})^{90}$, la Loi unifiée d'élargissement de la protection sociale en faveur des ressortissants des pays membres du GCC travaillant dans un autre Etat membre. Comme souligné par AISS, «cette loi a eu des répercussions positives, avec notamment une extension de la couverture, le renforcement de la mobilité des salariés et le développement d'une plus grande coopération économique entre les pays» ${ }^{91}$.

En Amérique latine, la Communauté Andine ${ }^{92}$ d'une part, et le Mercosur ${ }^{93}$ d'autre part, ont également adopté des accords de sécurité sociale. La déclaration adoptée par la Communauté Andine vise à remédier aux limites qui existent dans la couverture sociale des travailleurs migrants ainsi qu'à améliorer la coordination entre les régimes nationaux. Le Mercosur a conclu un accord en 2004 qui repose sur les principes d'égalité de traitement, de maintien des droits acquis et des droits en cours d'acquisition, et d'exportation des bénéfices. Il a développé un appareil législatif relativement sophistiqué par rapport à la Communauté Andine. Il vise à favoriser une réelle intégration au niveau régional grâce à l'introduction du statut de citoyen, absente dans le contexte andin ${ }^{94}$. Les pays membres de ces blocs régionaux se sont efforcés de garantir l'accès non discriminatoire aux migrants infrarégionaux et de faire en sorte que leurs prestations soient transférables. En outre, en 2011, la Convention ibéro-américaine de sécurité sociale est entrée en vigueur. Elle vise à garantir les prestations sociales en

\footnotetext{
${ }^{85}$ Dupper, 2014, p. 16 ; Pour une introduction aux différentes initiatives présents en Afrique, voir AISS, 2014, p. 60.

${ }^{86}$ Dupper, 2014, p. 11.

${ }^{87}$ Sabates-Wheeler etKoettls, 2010, p. 144.

${ }^{88}$ Dupper, 2014, p. 19.

${ }^{89}$ Forteza, Alvaro (2008), "The Portability of Pension Rights: General Principles and the Caribbean Case”, SP Discussion Paper No. 0825. Washington DC: World Bank.

${ }^{90}$ L'Arabie saoudite, le Royaume du Bahreïn, les Emirats arabes unis, le Koweït, Oman et le Qatar.

${ }^{91}$ AISS, 2014, p. 62.

${ }^{92}$ Décision 583 (2004) qui comporte des dispositions permettant l'exportation des prestations, le maintien des droits acquis et l'égalité de traitement.

${ }^{93}$ Accord multilatéral sur la sécurité sociale (1997). L’AISS mentionne cet accord comme cas exemplaire d'une " administration efficace » grâce à la mise en place d'un système informatique qui s'applique soit au niveau régional, soit au niveau bilatéral pour les pays concernés. AISS, 2014, p. 45.

${ }^{94}$ Bernal, Prada, et Uruena, 2015.
}

e-Revista Internacional de la Protección Social, ISNN 2445-3269. 2017, Vol. II, Nº 2

http://dx.doi.org/10.12795/e-RIPS.2017.i02.04.

Página 33 
matière d'invalidité, de vieillesse et de maladies professionnelles, et à mettre en place des mécanismes de coordination plus efficaces et effectifs ${ }^{95}$.

Par rapport au contexte européen, il convient de ne pas surestimer la portée de ces instruments. En effet, bien que leur élaboration illustre la volonté de fournir une réponse commune à la protection des travailleurs migrants, leur mise en œuvre démontre souvent une pratique encline à favoriser également l'adoption d'autres instruments au niveau national ou bilatéral qui fragmentent une approche d'intégration régionale.

\section{CONCLUSION}

Cette contribution a permis de mettre en lumière certains défis qui persistent en matière de protection sociale des travailleurs migrants. Tout d'abord, le risque majeur est qu'ils ne bénéficient pas pleinement de leurs cotisations. Des restrictions persistent en matière d'accès aux mécanismes de protection sociale, et ces limitations peuvent concerner les conditions d'accès (liées à la nationalité ou à la résidence) et varier selon les catégories de migrants. De plus, l'absence d'accords bilatéraux et/ou multilatéraux affecte la question de la portabilité des prestations.

L'évolution des mécanismes régionaux et multilatéraux dans le contexte de politique migratoire a eu un impact positif sur la façon de traiter la question de la protection sociale et de la transférabilité des droits acquis. La coordination supranationale est allée beaucoup plus loin que la voie bilatérale. Les conventions bilatérales peuvent masquer une attitude restrictive et sélective des Etats parties qui évoluent en parallèle avec les politiques migratoires au niveau national. Les Etats contractants peuvent identifier les bénéficiaires du droit à l'égalité, les branches de la sécurité sociale à inclure et les restrictions envisageables.

En conclusion, il semble indiscutable que le thème de la protection sociale des travailleurs migrants est strictement lié aux transformations en cours du rôle de l'Etat, associées aux questions migratoires ou de la libre circulation de personnes, et à l'avènement de la mondialisation. D'une part, la protection sociale est assurée par des modes de gouvernance associés à l'évolution de la gestion de flux migratoires, à travers des approches bilatérales ou régionales. Ce qu'il convient d'appeler la gouvernance néolibérale ne signifie pas pour autant moins d'Etat dans la gouvernance de la migration mais un changement avec des formes d'intervention étatique différentes, garanties par l'adoption de nouveaux instruments normatifs. D'autre part, cette transformation est aussi associée à l'évolution de l'accès des travailleurs migrants à la protection sociale qui met l'accent sur une dénationalisation progressive des processus de sauvegarde. En outre, l'absence d'une vision globale semble plutôt augmenter la fragilité normative dans ce domaine. Ceci impose aux travailleurs migrants un système complexe et peu compréhensible qui les fait vivre dans la crainte d'être privés des cotisations versées et des droits acquis dans leur pays d'accueil. Ce «bricolage» législatif risque d'augmenter

\footnotetext{
${ }^{95}$ Arellano Ortiz, 2013; Sánchez-Rodas Navarro, Cristina (2015), "Sinopsis del Reglamento 883/2004 y del Convenio Multilateral Iberoamericano de Seguridad Social dans Sánchez-Rodas Navarro, Cristina et Garrido Pérez, Eva (Dir.), El derecho del trabajo y la seguridad social: retos para la disciplina laboral ; Sánchez-Rodas Navarro, Cristina (2011), El Convenio Multilateral Iberoamericano de Seguridad Social", No 26 Revista General de Derecho del Trabajo y de la Seguridad Social.
}

e-Revista Internacional de la Protección Social, ISNN 2445-3269. 2017, Vol. II, Nº 2

http://dx.doi.org/10.12795/e-RIPS.2017.i02.04.

Página 34 
la confusion, ce qui conduit à repenser les termes de la question d'un point de vue juridique. 